\title{
Southern California Hypocenter Relocation with Waveform Cross- Correlation, Part 1: Results Using the Double-Difference Method
}

\author{
by Egill Hauksson and Peter Shearer
}

\begin{abstract}
We present the results of relocating 327,000 southern California earthquakes that occurred between 1984 and 2002. We apply time-domain waveform cross-correlation for $P$ and $S$ waves between each event and 100 neighboring events identified from the catalog based on a $3 \mathrm{D}$ velocity model. To simplify the computation, we first divide southern California into five polygons, such that there are $\sim 100,000$ events or less in each region. The polygon boundaries are chosen to lie in regions of sparse seismicity. We calculate and save differential times from the peaks in the cross-correlation functions and use a spline interpolation method to achieve a nominal timing precision of $0.001 \mathrm{sec}$. These differential times, together with existing $P$ - and $S$-phase picks, are input to the double-difference program of Waldhauser and Ellsworth $(2000,2002)$ to calculate refined hypocenters. We divide the southern California region into grid cells and successively relocate hypocenters within each grid cell. The overall resulting pattern of seismicity is more focused than the previously determined pattern from 1D or 3D models. The new improved locations are more clustered, in many cases by a factor of two or three, and often show clear linear alignments. In particular, the depth distribution is improved and less affected by layer boundaries in velocity models or other similar artifacts.
\end{abstract}

\section{Introduction}

The Southern California Seismic Network (SCSN) records about 12,000 to 35,000 earthquakes each year. These earthquakes form spatial patterns that remain almost stationary from year to year unless there are major mainshockaftershock sequences. In some areas, this pattern is well correlated with late Quaternary faults such as along the San Jacinto fault. In other cases, in contrast, there seems to be no correlation between the microseismicity and the fault structure. Sometimes this lack of correlation is attributed to mislocated hypocenters forming fictitious spatial patterns; thus, improving hypocenter accuracy is an important goal.

This article and its companion paper by Shearer et al. (2005) present results of a collaboration between University of California at San Diego and California Institute of Technology to improve the locations of southern California earthquakes by using differential times obtained by waveform cross-correlation. Here, we describe the cross-correlation calculations, the application of the double-difference method of Waldhauser and Ellsworth $(2000,2002)$, and some preliminary interpretation of the results. Shearer et al. (2005) present the results of applying the source-specific stationterm method and cluster analysis to relocate the same events. In general, the two methods produce very similar locations, which provides an important check on our results. However, there are some differences in the absolute locations related to differences in the seismic velocity models used for the calculations. In addition, the cluster analysis highlights the similar event clusters that include about $65 \%$ of the dataset and often provide very tight seismicity alignments. In contrast, the double-difference method rejects only about $10 \%$ of the data for our chosen parameters and provides a consistent overview of both clustered and scattered seismicity across southern California. Both earthquake catalogs are being made available to researchers, and these two articles constitute a preliminary interpretation of the improved catalogs and provide the necessary documentation to make the catalogs useful to other researchers (Hauksson et al., 2004).

Application of the double-difference algorithm to the more than 325,000 earthquakes in the SCSN catalog from 1984 to 2002 was achieved by dividing the southern California seismicity into grid cells and relocating the events in each grid cell sequentially. The major results of this effort are significantly sharper seismicity patterns than those determined with a $1 \mathrm{D}$ crustal velocity model and phase picks only. The relocated hypocenters are more tightly clustered with individual features often shrinking in size by a factor of two or three. The improved clustering makes it easier to interpret the seismicity patterns and to visually correlate them with mapped faults. The purpose of this article is to document the new seismicity catalog that is being made 
available to interested users via the Web from www. data.scec.org.

\section{Phase and Waveform Data Preprocessing}

We obtain phase and waveform data from the SCSN, as archived at the Southern California Earthquake Center (SCEC) Data Center for all available earthquakes and other events from 1984 to 2002 . We use the Seismic Transfer Protocol (STP) program (www.data.scec.org/STP/STPdocumentation) to extract the phase data (in ascii format) and the waveforms (in Seismic Analysis Code [SAC] format, www.llnl.gov/sac). We obtain all components (e.g., vertical, east, west) and channels (short-period, broadband, etc.) contained in the database archive. Because the resulting SAC files generally contain longer time windows than are necessary to include the $P$ - and $S$-wave phases, our first processing step is to trim the seismograms to $60 \mathrm{sec}$, starting $10 \mathrm{sec}$ before the theoretical $P$-arrival time. We store the resulting time series using the event-based Guy File System (GFS) format (G. Masters, personal comm., 2004) within a year/month directory structure. Following these steps, the GFS files consume about $500 \mathrm{~Gb}$ on an online Redundant Array of Independent Disks (RAID) system that provides rapid and random access to the data.

Because waveform cross-correlation relies on the similarity of waveforms between nearby events as recorded at the same station, it is important to have unique and consistent station identifiers. The same station name has sometimes been used on more than one network in southern California, so it is necessary to use the two-letter network identification code and the station name to be sure that stations are uniquely named. Thus, the program that converts the data to the GFS format also checks for consistency in network identification code, station name, and station location information. This ensures that the information in the GFS headers is consistent with a master list of station locations also generated at this time.

Our waveform cross-correlation algorithm requires that the data have the same sample rate, but the SCSN network has operated at several different sample rates during this period (most commonly, $50 \mathrm{~Hz}, 62.5 \mathrm{~Hz}, 80 \mathrm{~Hz}$, and 100 $\mathrm{Hz}$ ). In addition, we have learned that the cross-correlations are more reliable if the seismograms are bandpass filtered to between 1 and $10 \mathrm{~Hz}$. Thus, the next processing step resamples the data to a uniform $100-\mathrm{Hz}$ sample rate (using a spline interpolation method) and then applies a bandpass filter. To avoid having to store the complete processed dataset and to reduce the size of the problem in subsequent steps, we perform this operation just before running the cross-correlation on regionalized subsets of the event catalog. We define these regions in terms of polygons whose boundaries are chosen to avoid areas of dense seismicity (Fig. 1). There is some overlap in the polygon boundaries, and a few events can be in more than one polygon.

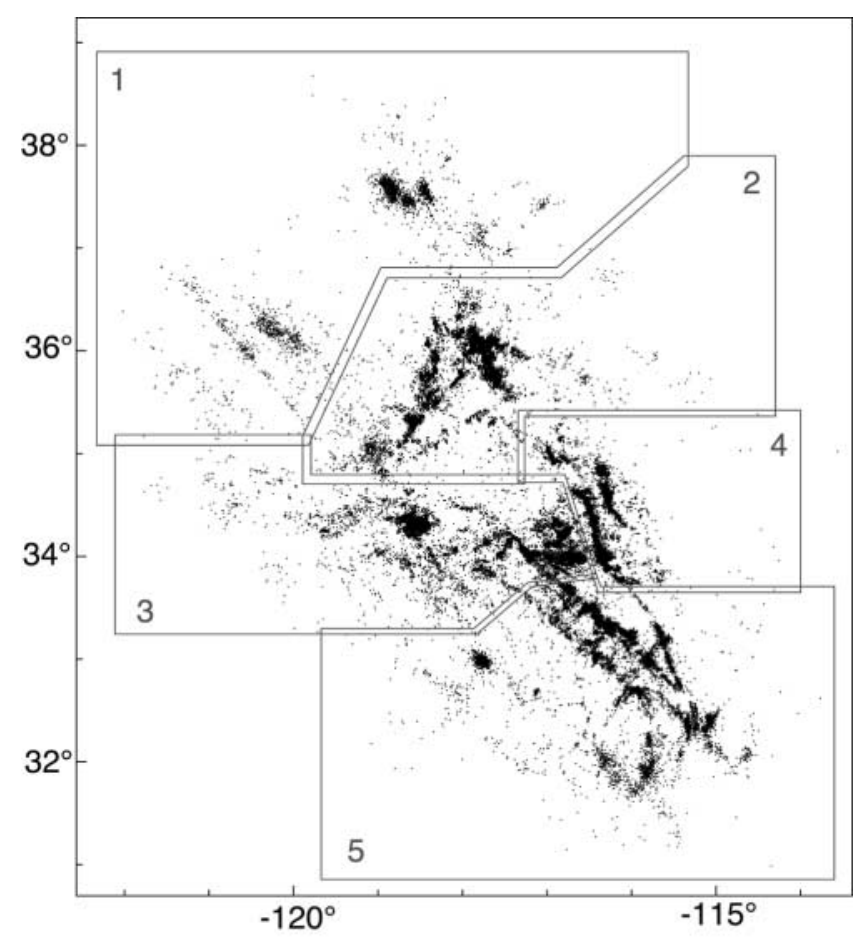

Figure 1. Boundaries of the five polygons used to divide southern California seismicity into subsets for easier processing. Polygon boundaries overlap slightly and are chosen to avoid the densest earthquake clusters.

\section{Waveform Cross-Correlation Calculation}

It would be numerically impractical to cross-correlate every event with every other event in the case of $\sim 340,000$ events because the size of the problem scales as $n(n-1) /$ 2 . Thus, we restrict the calculation to that between each event and 100 neighboring events. We use the Delaunay tesselation method for finding nearest neighbors (described in Astiz and Shearer, 2000), rather than simply the closest 100 events, to ensure that all events are "connected" in the calculations. We define the nearest neighbors using the event catalog based on the 3D velocity model of Hauksson (2000).

We compute the cross-correlation functions separately for $P$ and $S$ waves using a 2 -sec window around $P$ and a 3 sec window around $S$ and applying time shifts of up to \pm 1.5 sec, using a spline interpolation method to achieve a nominal timing precision of $0.001 \mathrm{sec}$. These time windows are defined by using computed arrival times for $P$ and $S$, referenced to a $1 \mathrm{D}$ gradient-velocity model, because often picks are not available to define the arrivals more precisely. In practice, we find for the southern California data that these predicted arrivals are within about $0.5 \mathrm{sec}$ of the true arrivals observed on the seismograms, provided that the event locations and origin time are consistent with the velocity model used in the calculation. An example of the cross-correlation calculation is shown in Figure 2 for two aftershocks of the $M 5.4$ Big Bear, California, earthquake of 22 February 2003. In 

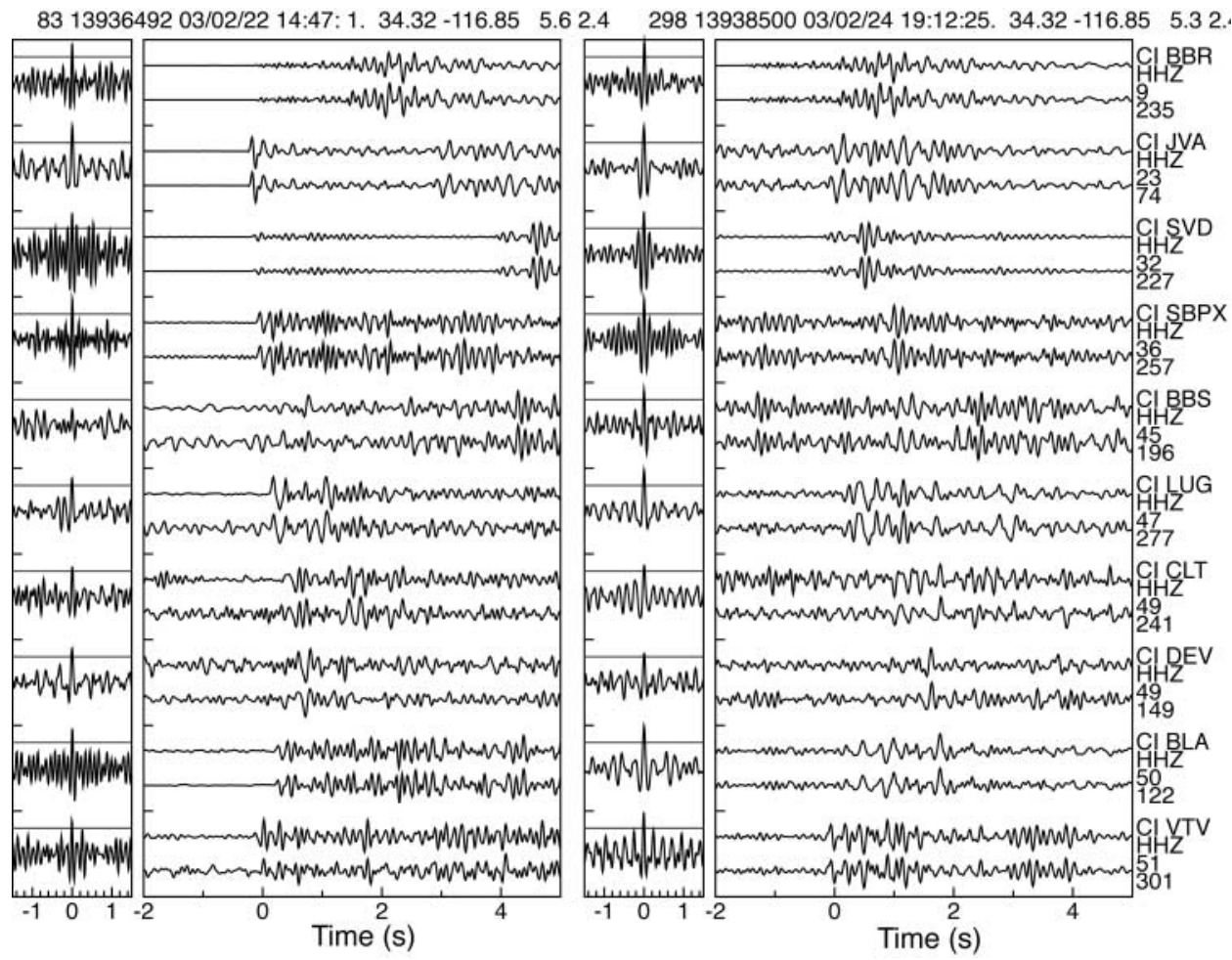

Figure 2. Waveforms and cross-correlation functions for two $M 2.4$ aftershocks of the 22 February 2003, M 5.4 Big Bear earthquake. Vertical-component traces from ten stations are shown, aligned on the theoretical $P$ arrival (left) and $S$ arrival (right). Labels to the right indicate the station name, channel, source-receiver distance $(\mathrm{km})$, and azimuth (in degrees at source to receiver). The plots to the left of the waveforms show the cross-correlation function for the two events at the given station; the horizontal line shows the level of a 0.6 correlation coefficient. This pair of events is similar enough that most stations have a well-defined peak in the cross-correlation function, which can be used to provide accurate relative timing information between the events (see text).

this case, the waveforms are very similar, but not identical, for both the $P$ and $S$ waves.

Information is saved only for those event pairs with an average waveform correlation coefficient of 0.45 or greater and with at least 10 individual differential times with correlation coefficients of 0.6 or greater. We separately perform the waveform cross-correlation for all channels that are available for each station. For each $P$ and $S$ measurement, we save the peak correlation coefficient, the time shift at the peak, the best-fitting amplitude scaling between the traces at the peak, and the position of the first negative sidelobe to the peak (useful for discriminating against spurious correlations). We also save results for the peak negative correlation (potentially useful to sort out instrument polarity flips and/or differential focal mechanisms), although we have not yet used this information. We use only positive correlations because in our experience similar event waveforms are almost always positively correlated and this minimizes the possibility of half-cycle errors at the cost of occasionally preventing valid negative correlations in the case of instrument polarity errors or differing focal mechanisms.

The resulting correlation coefficients and time shifts be- tween event pairs contain information regarding the similarity of events and their relative locations. A variety of different methods can be applied to analyze cross-correlation data. This article applies the double-difference method (Waldhauser and Ellsworth, 2000, 2002); the accompanying paper by Shearer et al. (2005) uses a cluster analysis method.

\section{Application of the Double-Difference Method}

These differential times, together with existing $P$ - and $S$-phase picks, are input to the hypoDD computer code of Waldhauser (2001) to calculate refined hypocenters. At this stage we exclude quarry blasts and other artificial events that are flagged in the SCSN catalog to focus on natural seismicity. Because hypoDD uses a 1D velocity model and relative locations of closely spaced events are not strongly dependent on the velocity model, we used a layered velocity model derived by Hauksson et al. (1995), which is similar to the Hadley and Kanamori (1977) model (figure 1 in Shearer et al., 2005). The starting locations were determined using the 3D $V_{p}$ and $V_{p} / V_{s}$ models of Hauksson (2000), which previ- 
ously were determined by using the SIMULPS computer code from Thurber (1993).

To investigate if poorly modeled velocity structure could affect our absolute hypocenters, as suggested by Wolfe (2002) and Michelini and Lomax (2004), we compared the Hauksson (2000) hypocenters with two sets of double-difference hypocenters determined for about 10,000 earthquakes in the southern Sierra Nevada. The first set of double-difference hypocenters was determined by using the grid-cell approach, whereas the second was determined independently for the whole region. All three sets of hypocenters coincide in map view, thus confirming that there are no systematic offsets between the three datasets. Further, the double-difference hypocenters exhibit stronger clustering of small clusters or epicenter alignments within the distribution than the hypocenters determined with the $3 \mathrm{D} V_{p}$ and $V_{p} / V_{s}$ models (Hauksson, 2000). Thus, the double-differencedetermined hypocenters appear to have no biases related to the use of the grid-cell method or poorly modeled velocity structure.

The phase picks are preprocessed to select data from connected events by using a technique developed by Waldhauser (2001). Using this technique, we built a network of links between events to form a chain of pairwise connected events. We selected a maximum hypocentral separation of $15 \mathrm{~km}$ and a minimum number of seven links to define a neighbor. If more conservative values are selected for these parameters, a significant number of events will not cluster and thus are not included in the hypoDD relocations. The preprocessing is also used to remove delay-time outliers that are much larger than the expected value for the respective event pair. The hypoDD code continues to refine the clustering through selection of a maximum distance between linked pairs. In each hypoDD run, we systematically decreased the maximum distance between linked pairs from $10 \mathrm{~km}$ to $5 \mathrm{~km}$ for phase data, and $9 \mathrm{~km}$ to $2 \mathrm{~km}$ for crosscorrelation data.

We used all available phase picks and cross-correlation differential travel times for each event in the relocation process. First, we relocated each event by using two iterations that include the phase picks with a weight of 1.0, a residual cutoff of four times the standard deviation, and differential travel times with a weight of 0.01 . Only two iterations were chosen to preserve the high-quality hypocenters from the 3D $V_{p}$ and $V_{p} / V_{s}$ models. Second, we continued the relocation process for 15 iterations, where the phase picks were given a weight of 0.01 and the differential travel times are given a weight of 1.0. Each differential travel-time measurement is weighted with the square of the cross-correlation coefficient. After ten iterations with the differential travel times, a residual cutoff of four times the standard deviation was applied to remove outliers. A single damping factor was selected for all the grid cells after several tests were done with the Vallecitos Valley cluster and the most common dataset size. However, because the damping depends on the number of data in each inversion, the damping may not always be optimal for all the grid cells, which can in some cases lead to less dense clustering of events than if an optimal damping value was chosen through experimentation. This approach was determined to give the best results through testing based on data from the alignment of earthquakes in Vallecitos Valley as shown in figure 3 of Shearer et al. (2005).

Because we can only fit travel-time data from a maximum of $\sim 12,000$ earthquakes into each hypoDD inversion, we divided the southern California seismicity into $1850 \mathrm{rec}-$ tangular grid cells, extending from $32^{\circ}$ to $37.4^{\circ}$ and $114.15^{\circ}$ to $121.5^{\circ}$. Each grid cell is $0.30^{\circ}$ in latitude and $0.30^{\circ}$ in longitude. We use a simple grid technique (A. Michael, written comm., 2001) to step through the region in steps of $0.15^{\circ}$ of latitude and longitude and relocate the earthquakes within each grid cell. Because the grid cells overlap 50\%, the hypocenter of each earthquake is calculated at least four times and the final hypocenter is a weighted average of the available hypocenters. The weight assigned to each hypocenter is proportional to the distance of the hypocenter away from the center of the grid cell. If a grid cell has more than 12,000 events, it is subdivided into smaller subcells. Each grid cell has data from 2000 earthquakes in common to ensure that the event clustering is not being adversely affected by the grouping of the data. Because in these cases the same earthquake can be located several times, each earthquake is assigned a weight inversely proportional to its distance from the center of the grid cell. In the last step of the processing, the final solution for each event is calculated as the weighted average of all the available solutions for the respective event.

The hypoDD program does not estimate hypocentral errors directly except for small datasets and it is not feasible to carry out detailed error estimates for large datasets. The absolute errors are best evaluated with the 3D velocity model. Hauksson (2000) showed the average absolute errors for this dataset are $1.5 \mathrm{~km}$ in the horizontal and $3.0 \mathrm{~km}$ in the vertical direction. Comparison of the width of the epicentral distributions in the Vallecitos Valley cluster determined with four different methods (SCSN catalog, sourcespecific station terms, double-difference method, and cluster method) can also be used to get an independent estimate of the hypocental errors (figure 3 in Shearer et al. [2005]). If we presume that most of those hypocenters follow a planar surface, the horizontal width of the alignment of epicenters should be a measure of the uncertainty. The hypocenters determined by using a 1D model show horizontal errors of $\pm 2.0 \mathrm{~km}$ and vertical errors of $\pm 4.0 \mathrm{~km}$ when assuming that the vertical errors are about twice as large as the horizontal errors. The hypoDD-determined hypocenters provide a factor of two in improvement and have horizontal errors of less than $\pm 0.5 \mathrm{~km}$ and vertical errors of less than \pm 2.0 $\mathrm{km}$. These error estimates are consistent with published relative errors from hypoDD, when differential travel times are used, which are on the order of tens to a few hundreds of meters (Waldhauser and Ellsworth, 2001).

The relocated catalog from 1984 to 2002 is available at www.data.scec.org. We have combined both hypoDD 
(306,657 events) and locations determined by using 3D velocity models $(20,773)$ to assemble a complete catalog, because hypoDD may reject certain hypocenters if they do not meet the criteria of being part of a cluster. This catalog excludes most man-made explosions and is well suited for analyzing spatial and temporal seismicity patterns.

\section{Results: Seismicity Patterns}

The hypocenters in the new catalog of hypoDD exhibit improved clustering both horizontally and vertically, creating a more focused picture of the complex distribution of seismicity (Fig. 3). In general, the overall distribution of these hypocenters coincides with the hypocenters determined by Hauksson (2000) using the 3D $V_{p}$ and $V_{p} / V_{s}$ models. The overall distribution of the seismicity maintains its spatial relationship to the major late Quaternary faults, with the bulk of the seismicity forming clustered distributions adjacent to the faults. In particular, the San Jacinto fault trace extends through an almost symmetric distribution of hypocenters forming sharp alignments that in most cases are adjacent to, but not coincident with, the mapped surface traces.
These alignments are either parallel to the traces or form high angles to them.

The seismicity around the southern San Andreas fault shows clear alignment along the southern part of the Carrizo Plain segment, whereas both the Mojave and Coachella Valley segments are dominated by off-fault hypocenters. Extending from Banning Pass to San Bernardino, a sharp northern edge of the seismicity distribution strikes a few degrees north of west, cutting across the main trace of the San Andreas fault at a shallow angle. Earthquake swarms in the Salton Sea at the south end of the San Andreas fault suggest the presence of two north-northwest-striking seismic zones.

In many cases the small earthquakes may not be occurring near the surface traces of the major late Quaternary faults but rather reflect brittle deformation adjacent to these faults. Several northeast-trending seismicity alignments do not coincide with any nearby late Quaternary faults, such as the seismicity in the area between the San Jacinto and Elsinore faults. Sometimes the late Quaternary faults appear to bracket both the alignments and 3D scattered distributions, with higher levels of seismicity on one side, such as to the east of the Elsinore and to the north of the Hollywood-Santa Monica faults.

\section{Southern California Seismicity 1984 - 2002}

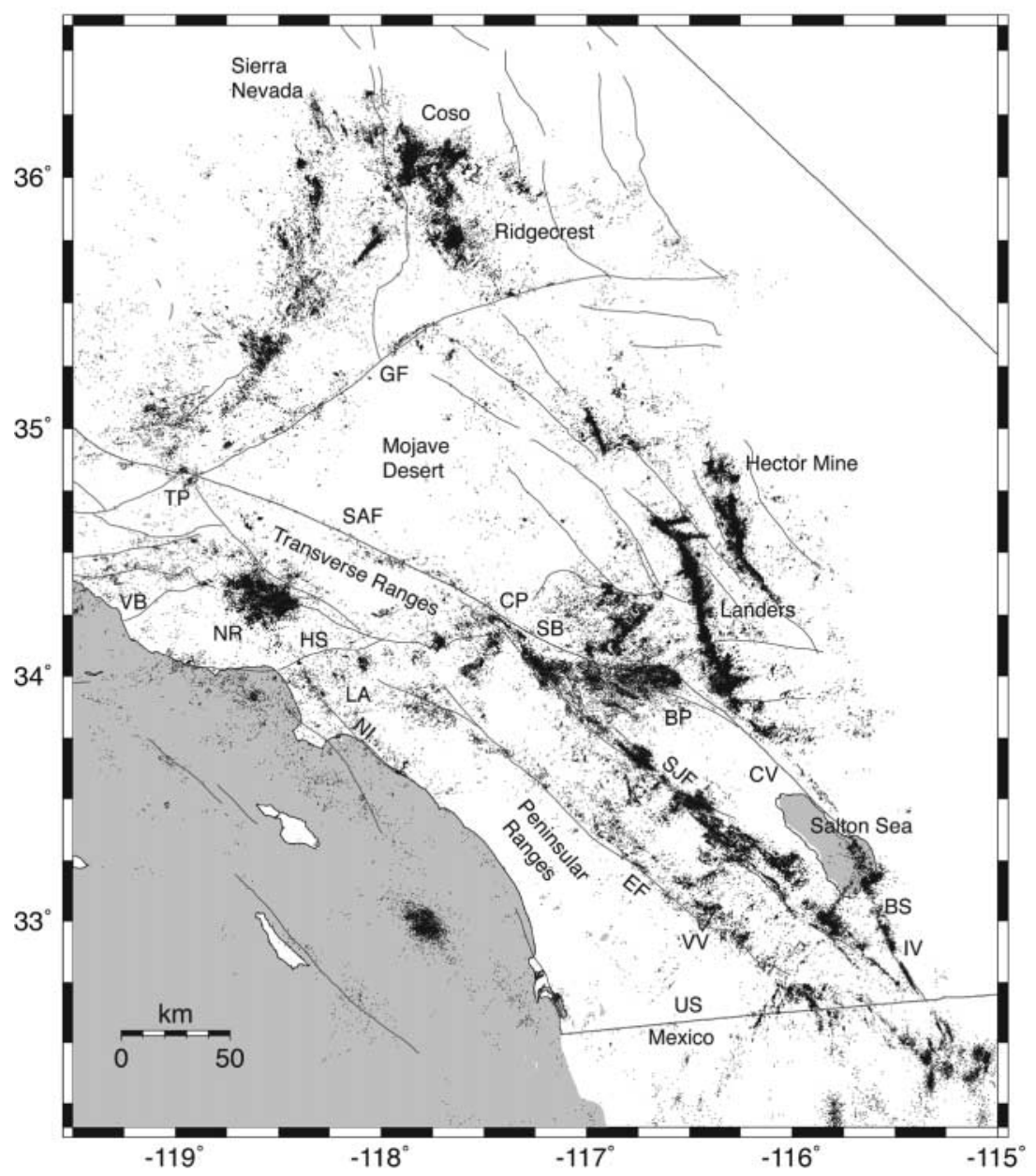

Figure 3. The relocated southern California seismicity $1984-2002$ by using the doubledifference method. BP, Banning Pass; BS, Brawley seismic zone; CP, Cajon Pass; CV, Coachella Valley; EF, Elsinore fault; GF, Garlock fault; HS, Hollywood-Santa Monica fault; IV, Imperial Valley; LA, Los Angeles; NI, Newport-Inglewood fault; NR, Northridge; SAF, San Andreas fault; SB, San Bernardino Mountains; SJF, San Jacinto fault; TP, Tejon Pass; VB, Ventura Basin; VV, Vallecitos Valley. 
In the Los Angeles basin, the seismicity has always exhibited a scattered 3D distribution that has sometimes been portrayed as an artifact of the complex 3D velocity structure. Individual seismicity clusters are more compact but still form an apparent random scatter in the basin. Because all the relocated hypocenters exhibit this scattered 3D distribution, we conclude that the scatter is real and reflects a very complex 3D brittle deformation along interlaced strike-slip and thrust faults (Hardebeck and Hauksson, 2001). Within the 3D cloud of seismicity, the hypocenters along the Newport-Inglewood fault form a sharp north-trending alignment along the northernmost part of the fault and a diffuse distribution to the south, where the 1933 Long Beach earthquake occurred. Similarly, several clusters and scattered background seismicity extend from east to west across the basin and illuminate the blind thrusts beneath the north edge of the basin.

The major aftershock sequences, the 1992 Landers, 1994 Northridge, and 1999 Hector Mine, form clusters, have distinct internal structures illuminating the mainshock rupture surface, and in some cases have complex secondary faults. Some of these alignments suggest that high-angle cross-faults were activated by the mainshock.

The depth distribution of the seismicity varies spatially across southern California (Fig. 4). The depth patterns of the seismicity within the thrust-faulting region of the Los Angeles basin and the Ventura basin are diffuse. The Elsinore and San Jacinto faults exhibit more mature seismicity patterns and, in particular, the San Jacinto fault shows enhanced levels of seismicity around the brittle-ductile transition zone (Nazareth and Hauksson, 2004). The noticeable vertical streaks along the San Jacinto fault and the southern Elsinore fault support the idea presented by Shearer et al. (2005) of cross-faults defined by seismicity, which in many cases intercept the late Quaternary faults at a high angle.

Background seismicity is often used to measure the thickness of the seismogenic crust. Relocating the southern California seismicity by using 3D models had a significant

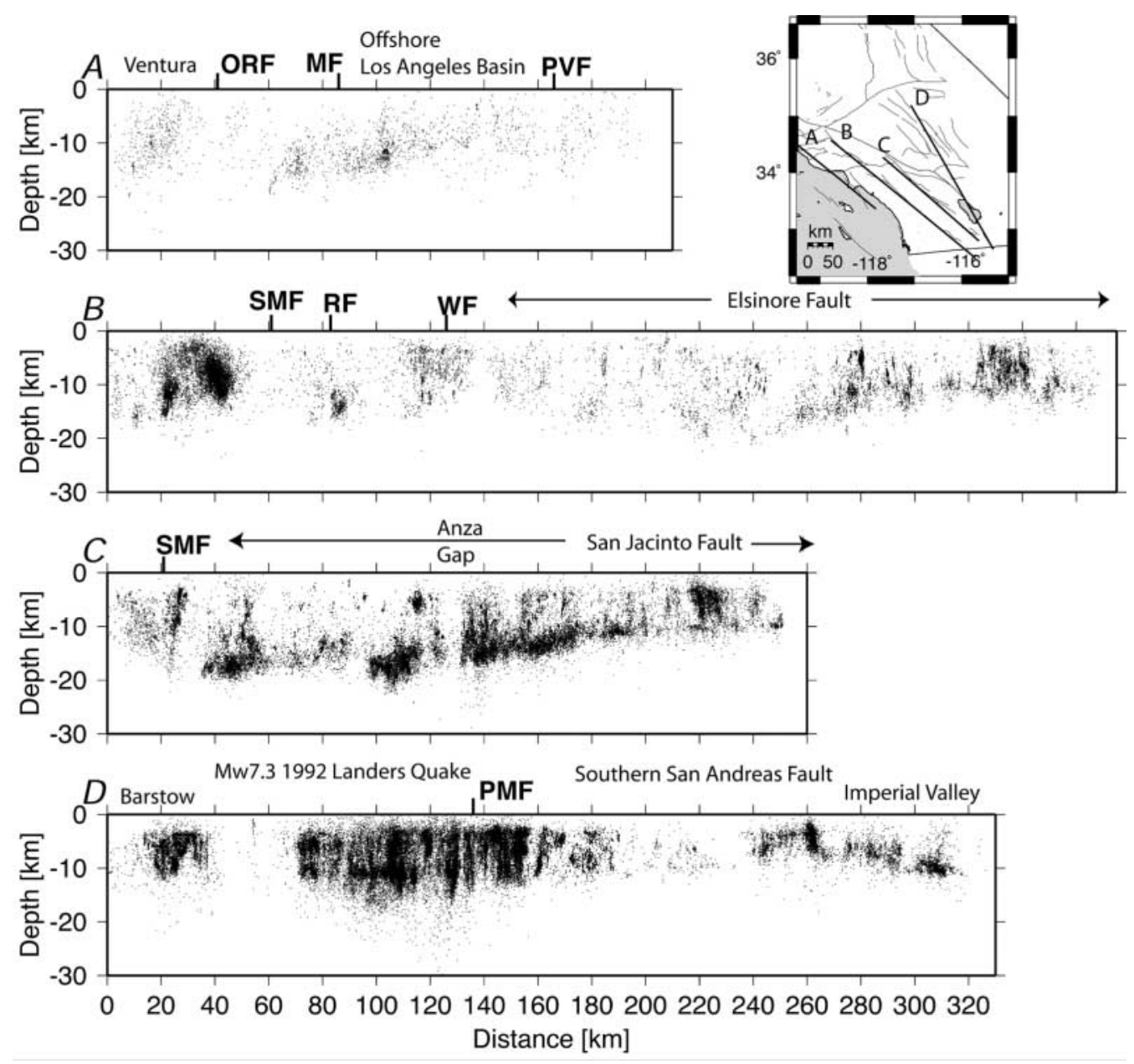

Figure 4. Cross sections that include a 10-km-wide band of seismicity along profiles shown in the inset map. Similar depth profiles were also published by Hill et al. (1990). ORF, Oakridge fault; MF, Malibu fault; PVF. Palos Verdes fault; SMF, Sierra Madre fault; RF, Raymond fault; WF, Whittier fault; PMF, Pinot Mountain fault. See Figure 2 for more details about abbreviations. 
effect on the depth distribution (Nazareth and Hauksson, 2004). The double-difference relocations provide improved clustering but do not change the absolute depth distribution, as expected. The decrease in depth of the seismicity toward the Salton Sea area is often associated with the high heat flow in this region (Nazareth and Hauksson, 2004), whereas the increase in depth beneath the central Peninsular Ranges remains an enigma.

\section{Discussion}

The southern California seismicity patterns have been thought to suffer from various degrees of mislocations in the past. For instance, the linear cluster of seismicity adjacent to the southern San Andreas fault in Coachella Valley was thought to be artificially offset from the main trace of the San Andreas fault because of the low velocities in Coachella Valley (Fig. 3). By using starting hypocenters that take into account the 3D velocity model, we have removed the velocity model bias and the hypocenters are still offset from the mapped trace of the San Andreas fault. Thus, the new catalog validates many of the intriguing but unexplained features of southern California seismicity.

Relocating this large dataset of more than 327,000 earthquakes was challenging because the analysis of the waveforms and parametric datasets is a computationally intensive task. The Seismic Transfer Program (STP) interface at SCEDC made this project possible because the waveforms and parametric data were downloaded automatically. To accomplish the processing we had to make selections such as deciding the number of nearest neighbors for cross-correlations, determining damping values, and selecting a regional velocity model for the double-difference relocations. Although our results could probably be improved by doing individual studies of limited areas by optimizing configuration parameters for each processing step, the results of this study provide a uniform look across all of southern California. Thus, the new catalog can be used to analyze spatial and temporal seismicity patterns across the whole region.

The accompanying article by Shearer et al. (2005) uses a cluster location technique and obtains similar results. A comparison of the different techniques is shown in figure 3 of Shearer et al. (2005). The results from both techniques can be improved further by using local velocity models or by optimizing the configuration parameters of each code for the specified region. For instance, using a local velocity model for the Imperial Valley, Shearer et al. are able to improve the resolution of the cross-faults that connect the southern tip of the San Andreas fault across the Salton Sea to the Brawley seismic zone.

Hill et al. (1990) analyzed seismicity from 1980 to 1986 across California. They provided a map view and cross sections similar to what we provide in Figures 3 and 4. When comparing the map view, the relocated seismicity exhibits denser clustering and in some cases new patterns have emerged. The most obvious new patterns are the $1992 M_{\mathrm{w}}$
7.3 Landers, $1994 M_{\mathrm{w}} 6.7$ Northridge, and $1999 M_{\mathrm{w}} 7.1 \mathrm{Hec}-$ tor Mine mainshock-aftershock sequences. The areas with a steady high rate of seismicity, such as Banning Pass and the whole length of the San Jacinto fault, remain as active today as they were two decades ago. The biggest difference between the Hill et al. (1990) and the present relocations can be seen in cross sections, where the relocated seismicity exhibits denser clustering and many artifacts, such as layering that corresponded to layer boundaries in the velocity models, have been removed. Similarly, the apparent seismicity at 0 to $1 \mathrm{~km}$ depth that appears in the cross section by Hill et al. merges to greater depths as a result of the relocation process.

\section{Conclusions}

The regional seismicity patterns in southern California are brought into focus when the hypocenters are relocated by using the double-difference method. In detail, often the spatial correlation between background seismicity and late Quaternary faults is improved as the hypocenters become more clustered, and the spatial patterns are more sharply defined. Along some of the strike-slip faults the seismicity clusters decrease in width and form alignments, implying that in many cases the clusters are associated with a single fault. In contrast, for instance, the Los Angeles Basin seismicity remains mostly scattered, reflecting $3 \mathrm{D}$ distribution of the tectonic compression.

\section{Acknowledgments}

The Southern California Seismic Network (SCSN), Kate Hutton and her staff, produced the earthquake phase picks used in this study, and the data were made available at the Southern California Earthquake Data Center. We thank Andy Michael of U.S. Geological Survey, Menlo Park, for making available his technique for relocating the catalog with the grid-cell method. We thank L. Jones, J. Hardebeck, and D. Schaff for reviewing the manuscript, and I. Wong for constructive editorial comments. The figures were done using GMT (Wessel and Smith, 1991). This work was supported by NEHRP/USGS Grant 04HQGR0052 and by SCEC, which is funded by NSF Cooperative Agreement EAR-0106924 and USGS Cooperative Agreement 02HQAG0008; SCEC contribution no. 820. Contribution no. 9105, Division of Geological and Planetary Sciences, California Institute of Technology, Pasadena.

\section{References}

Astiz, L., and P. M. Shearer (2000). Earthquake locations in the inner Continental Borderland, offshore southern California, Bull. Seism. Soc. Am. 90, 425-449.

Hadley, D. M., and H. Kanamori (1977). Seismic structure of the Transverse Ranges, California, Geol. Soc. Am. Bull. 88, 1461-1478.

Hardebeck, J. L., and E. Hauksson (2001). Crustal stress field in southern California and its implications for fault mechanics, J. Geophys. Res. 106, 21,859-21,882.

Hauksson, E. (2000). Crustal structure and seismicity distributions adjacent to the Pacific and North America plate boundary in southern California, J. Geophys. Res. 105, 13,875-13,903.

Hauksson, E., L. M. Jones, and K. Hutton (1995). The 1994 Northridge earthquake sequence in California: seismological and tectonic aspects, J. Geophys. Res. 100, 12,335-12,355. 
Hauksson, E., P. Shearer, and J. Vidale (2004). Converting advances in seismology into earthquake science, EOS Trans. A.G.U. 85, 3-8.

Hill, P. H., J. P. Eaton, and L. M. Jones (1990). Seismicity 1980-86, in the San Andreas fault system, California, U.S. Geol. Surv. Profess. Pap. $1515,115-152$.

Michelini, A., and A. Lomax (2004). The effect of velocity structure errors on double-difference earthquake locations, Geophys. Res. Lett. 31, L09602, doi 10.1029/2004GL019682.

Nazareth, J. J., and E. Hauksson (2004). The Seismogenic thickness of the southern California crust, Bull. Seism. Soc. Am. 94, 940-960.

Shearer, P. M., E. Hauksson, and G. Lin (2005). Southern California hypocenter relocation with waveform cross-correlation, Part 2: Results using source-specific station terms and cluster analysis, Bull. Seism. Soc. Am. 95, no. 3, 904-915.

Thurber, C. H. (1993). Local earthquake tomography: velocities and Vp/ Vs-theory, in Seismic Tomography: Theory and Practice, H. M. Iyer and K. Hirahara (Editors), Chapman and Hall, London, 563-583.

Waldhauser, F. (2001). hypoDD: a program to compute double-difference hypocenter locations (hypoDD version 1.0-3/2001), U.S. Geol. Surv. Open File Rept. 01-113.

Waldhauser, F., and W. L. Ellsworth (2000). A double-difference earthquake location algorithm: method and application to the northern Hayward fault, California, Bull. Seism. Soc. Am. 90, 1353-1368.
Waldhauser, F., and W. L. Ellsworth (2002). Fault structure and mechanics of the Hayward fault, California, from double-difference earthquake locations, J. Geophys. Res. 107, no. B3, doi 10.1029/2000JB000084.

Wessel, P., and W. H. F. Smith (1991). Free software helps map and display data, Eos Trans. AGU. 72, 441, 445-446.

Wolfe, C. J. (2002). On the mathematics of using difference operators to relocate earthquakes, Bull. Seism. Soc. Am. 92, 2879-2892.

Seismological Laboratory

California Institute of Technology

Pasadena, California 91125

hauksson@gps.caltech.edu

(E.H.)

Institute of Geophysics and Planetary Physics

Scripps Institution of Oceanography

University of California at San Diego

La Jolla, California 92093-0225

pshearer@ucsd.edu

(P.M.S.)

Manuscript received 23 August 2004. 